\title{
Identification of SNPs associated with muscle yield and quality traits using allelic- imbalance analyses of pooled RNA-Seq samples in rainbow trout
}

\author{
Rafet Al-Tobasei ${ }^{1}$, Ali Ali ${ }^{2}$, Timothy D. Leeds ${ }^{3}$, Sixin Liu ${ }^{3}$, Yniv Palti ${ }^{3}$, Brett Kenney ${ }^{4}$ and Mohamed Salem ${ }^{1,2^{*}}$ (i)
}

\begin{abstract}
Background: Coding/functional SNPs change the biological function of a gene and, therefore, could serve as "large-effect" genetic markers. In this study, we used two bioinformatics pipelines, GATK and SAMtools, for discovering coding/functional SNPs with allelic-imbalances associated with total body weight, muscle yield, muscle fat content, shear force, and whiteness. Phenotypic data were collected for approximately 500 fish, representing 98 families ( 5 fish/family), from a growth-selected line, and the muscle transcriptome was sequenced from 22 families with divergent phenotypes ( 4 low- versus 4 high-ranked families per trait).
\end{abstract}

Results: GATK detected 59,112 putative SNPs; of these SNPs, 4798 showed allelic imbalances ( $>2.0$ as an amplification and $<0.5$ as loss of heterozygosity). SAMtools detected 87,066 putative SNPs; and of them, 4962 had allelic imbalances between the low- and high-ranked families. Only 1829 SNPs with allelic imbalances were common between the two datasets, indicating significant differences in algorithms. The two datasets contained 7930 non-redundant SNPs of which 4439 mapped to 1498 protein-coding genes (with 6.4\% non-synonymous SNPs) and 684 mapped to 295 IncRNAs. Validation of a subset of 92 SNPs revealed 1) 86.7-93.8\% success rate in calling polymorphic SNPs and 2) 95. $4 \%$ consistent matching between DNA and cDNA genotypes indicating a high rate of identifying SNPs with allelic imbalances. In addition, 4.64\% SNPs revealed random monoallelic expression. Genome distribution of the SNPs with allelic imbalances exhibited high density for all five traits in several chromosomes, especially chromosome 9, 20 and 28. Most of the SNP-harboring genes were assigned to important growth-related metabolic pathways.

Conclusion: These results demonstrate utility of RNA-Seq in assessing phenotype-associated allelic imbalances in pooled RNA-Seq samples. The SNPs identified in this study were included in a new SNP-Chip design (available from Affymetrix) for genomic and genetic analyses in rainbow trout.

Keywords: Rainbow trout, Fish, SNPs, Genetic markers, RNA-Seq

\section{Background}

Fish growth rate, muscle yield and fillet quality are major traits affecting profitability of aquatic food animal production. As feed cost is a major factor influencing the profitability, efficiency of growth is important and related to growth rate and muscle yield and composition.

\footnotetext{
* Correspondence: Mohamed.salem@mtsu.edu

${ }^{1}$ Computational Science Program, Middle Tennessee State University, Murfreesboro, TN 37132, USA

${ }^{2}$ Department of Biology and Molecular Biosciences Program, Middle

Tennessee State University, Murfreesboro, TN 37132, USA

Full list of author information is available at the end of the article
}

Skeletal muscle constitutes about $50-60 \%$ of the fish weight [1]. Given that growth efficiency and fillet firmness and appearance are critical for profitability and production of premium products [2], optimizing fish growth, muscle yield and fillet quality traits is a key objective in aquaculture breeding programs. Traditional phenotype-based selection is typically used to select for fast growth; however, muscle yield and quality traits are difficult to improve by conventional selection because measurement of these traits requires sacrificing the animal [2]. 
Genomic selection tools have been created to improve economically important traits in plants and livestock. Genetic maps, which characterize the linkage or coinheritance patterns of genetic markers, have been developed for a wide range of species, including fish, with the aim of discovering allelic variation affecting traits; and ultimately identify DNA sequences underlying phenotypes [3, 4]. Markers have been identified by various molecular techniques, including numerous and genomewide single nucleotide polymorphisms (SNPs). In addition, recent technological developments have enabled high throughput genotyping of these SNPs rendering them useful for genome-wide association studies [5-8]. Functional SNPs are generally defined as SNPs from genome sequences that affect structure, expression or function of a gene. These sequences include coding SNPs (e.g. non-synonymous, splicing), promoter and noncoding SNPs, as well as functional elements identified from studying of genome conservation [9]. Coding/ Functional/ SNPs (c/fSNPs) are especially important because they have the potential to change the function of a protein $[4,10,11]$. In addition, c/fSNP markers, because they are located within expressed genes, they are unlikely to become unlinked from their associated genes due to genetic recombination. Therefore, c/fSNPs can be useful genetic markers for detecting significant associations with phenotypes. Understanding molecular mechanisms of muscle growth and quality can help in making better selection decisions. In terrestrial livestock, several genes, genetic markers and QTLs associated with production traits, including growth, have been characterized using molecular techniques $[12,13]$. In addition, marker-assisted selection has been used to enhance genetic improvement in livestock breeding programs by direct selection on genes affecting economic traits [14] and to optimize selection for quantitative traits $[12,13]$. However, the genetic basis of muscle growth and quality traits is not well studied in fish [15].

Rainbow trout is the most cultivated cool and cold freshwater fish in the U.S. [16], and it is considered a model species for studies in several fields of biology, including ecology [17], pathology [18], physiology [19], toxicology [20] and carcinogenesis [21]. Several studies used RNA sequencing to identify markers in human [22, 23] and non-model species [11, 24, 25]. However, most SNP detection algorithms were developed for DNA-Seq analyses and are not optimized/tested for RNA-Seq, especially in pooled samples. The objective of this study was using RNA-Seq analyses of pooled samples to identify c/fSNP markers and develop a resource for studies of marker association with production traits in rainbow trout. First, transcriptome-wide SNP allele frequencies were correlated to phenotypic variations in fish whole body weight (WBW) and muscle yield, fat content, shear force and whiteness. Second, SNPs with allelic imbalance scores (ratios between the allelic frequencies of the highend families and that of the low-end families) were identified. Then, a subset of the putative SNPs was validated for allelic polymorphism and tested for trait association. Finally, genes harboring SNPs with allelic imbalances were annotated to obtain insight into the potential functional effects of the SNPs.

\section{Result and discussion \\ Phenotypes}

SNPs were identified in fish families with divergent phenotypes in WBW, muscle yield, fat content, shear force (texture) and whiteness of the fillet. These rainbow trout were from a growth-selected line developed by the NCCCWA breeding program [26]. Briefly, this line was created through artificial selection, starting in 2004, from 7 founder strains with documented diversity and domestication history. Over five generations, the population responded to selection by $9.8-12.7 \%$ increase in WBW per generation, and rate of inbreeding averaged $0.86 \%$ per generation [26]. In this study population, which was sampled after three generations of selection (hatch year of 2010), WBW was positively correlated with muscle yield and muscle fat content $\left(R^{2}=0.56\right.$ and 0.50 respectively, data not shown). Our previous reports showed that fast growth may be genetically associated with improved muscle yield, paler fillets (affected by intramuscular fat content) and firmer texture [27]. The trait heritability estimates for muscle yield, muscle weight, WBW10, WBW13, carcass weight, fat percentage, shear force and fillet color were moderate to high $(0.31-0.81)[6,27]$. Those moderate to high heritability estimates imply that substantial additive genetic variation exist in the study population for growth and carcass traits.

For RNA sequencing, muscle samples were collected from 7 to 9 different full-sib families showing divergent phenotypes per trait (i.e. 3-5 high ranked families versus 3-5 low ranked families per trait). Five fish were sampled from each family. Divergent phenotypic attributes (Fig. 1) were statistically different $(P<0.01)$ : WBW $(1221.6 \mathrm{~g} \pm 84.25$ vs. $502.1 \pm 28.0 \mathrm{~g})$, muscle yield $(50.9 \% \pm 1.6$ vs. $43.3 \% \pm 2.3)$, muscle crude-fat $(9.24 \%$ \pm 1.2 vs. $4.77 \% \pm 1.3$ ), shear force (grams force/g of sample; $539.64 \pm 12.3$ vs. $310.01 \pm 49.2$ ), and fillet whiteness index $(44.7 \pm 0.8$ vs. $41.23 \pm 0.4)$ for high- vs. low-ranking groups, respectively. Means and standard deviations of these traits were calculated from the family averages.

\section{Identification of putative SNPs}

RNA pools from muscle tissues of 5 fish per family were used for RNA-Seq analyses. A total of 259,634,620 reads (100 bp single-end) were generated from 22 families at 


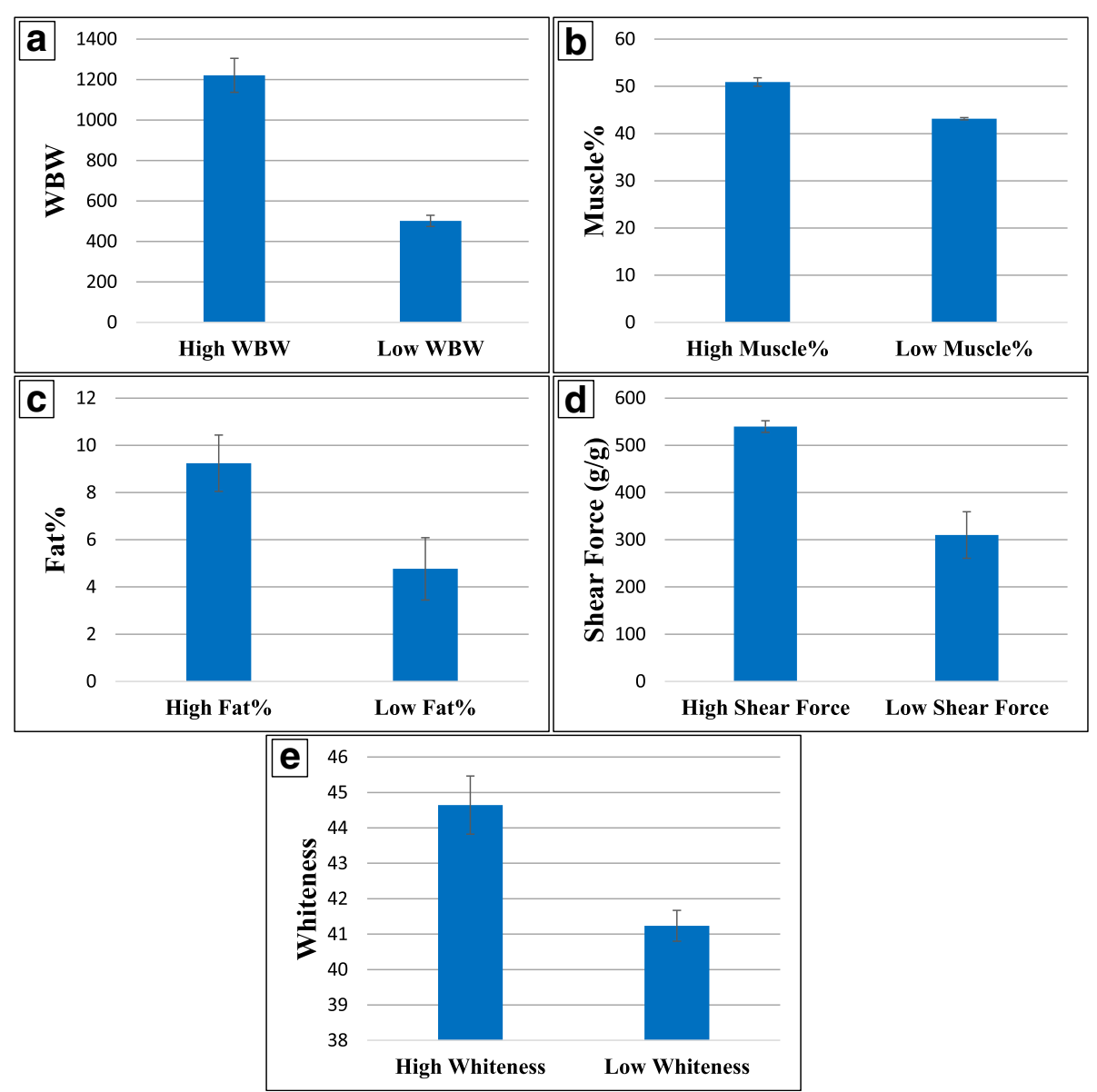

Fig. 1 Phenotypic variations in fish families with contrasting phenotypes for five different traits; whole-body weight (a), muscle yield (b), fat content $(\mathbf{c})$, shear force $(\mathbf{d})$ and fillet whiteness index $(\mathbf{e})$. All differences were statistically significant $(p<0.01)$

an average of $11,801,573$ reads per family. Reads were aligned against the rainbow trout genome [28] using the STAR [29] alignment tool. Percentage of reads mapped to the genome ranged from $80 \%$ to $82 \%$ per family.

A total of 204,604 putative SNPs were detected for the five traits using Haplotypecaller tool of Genome Analysis Toolkit v3.3.0 (GATK) [30], with an average of 40,920 SNPs per trait. Using the SAMtools/Popoolation software package [31, 32], a total of 304,805 putative SNPs were predicted, with an average of 60,961 SNPs per trait (Table 1). After removing redundant SNPs among all traits, we had 59,112 SNPs from GATK and 87,066 from SAMtools/Popoolation2 with 50,885 shared between the two bioinformatics pipelines (Table 1).

After identifying putative SNPs, an in-house Perl script was used to estimate allelic imbalances of the SNPs in each trait. A total of 6275 SNPs with allelic imbalances were identified from the GATK dataset at cutoff values of $>2.0$ as an amplification and $<0.5$ as loss of heterozygosity. In addition, 969 SNPs explicitly existed in only the high or low phenotypic group. After removing redundant SNPs between traits at the two cutoff values, there were 4798 unique SNPs (Table 1). Similarly, SAMtools/Popoolation2 identified 5070 SNPs with allelic imbalances at cutoff values of $>2.0$ as an amplification and $<0.5$ as loss of heterozygosity. In addition, 1450 SNPs existed in families at one of the two ends of each trait variation scale but not in the other (Table 1). There were 4962 non-redundant SNPs among the five traits that were identified with SAMtools/ Popoolation 2 at the two cutoff values. There were only 1829 non-redundant SNPs shared between GATK and SAMtools/Popoolation2. Differences in variant calling and filtering steps might have caused the observed differences in number of SNPs between GATK and SAMtools/Popoolation2. There were 7930 non-redundant SNPs with allelic imbalances from both methods. The results of the SNPs' allelic imbalances should be taken with caution because we could not find a reliable statistical test associated with the ratio calls derived from the allelic imbalance calculation to report statistical significance. However, by utilizing exact allele counts instead of frequencies, we were able to assign Chi Square $P$-Values to most of the SNPs with 
Table 1 Summary of putative SNPs and SNPs showing allelic imbalances identified by SAMtools and GATK for each trait

\begin{tabular}{|c|c|c|c|c|c|c|}
\hline \multirow[t]{3}{*}{ Trait } & \multicolumn{2}{|l|}{ No. of putative SNPS } & \multicolumn{4}{|c|}{ No. of SNPs with Allelic imbalance } \\
\hline & \multirow[t]{2}{*}{ SAMtools/Popoolation2 } & \multirow[t]{2}{*}{ GATK } & \multicolumn{2}{|c|}{ SAMtools/Popoolation2 } & \multicolumn{2}{|l|}{ GATK } \\
\hline & & & $0.5 / 2.0$ & $0.0 / 1.0$ & $0.5 / 2.0$ & $0.0 / 1.0$ \\
\hline Fat\% & 59,032 & 38,808 & 662 & 406 & 877 & 270 \\
\hline Shear & 60,309 & 38,960 & 910 & 488 & 1152 & 261 \\
\hline Muscle\% & 61,117 & 42,383 & 1321 & 116 & 1507 & 76 \\
\hline Whiteness & 64,636 & 44,460 & 1011 & 347 & 1283 & 298 \\
\hline WBW & 59,711 & 39,993 & 1166 & 93 & 1456 & 64 \\
\hline Total \# SNPS & 304,805 & 204,604 & 5070 & 1450 & 6275 & 969 \\
\hline Total \# SNPs non-redundant & 87,066 & 59,112 & 4962 & & 4798 & \\
\hline \multirow[t]{2}{*}{ Total Common SNPs } & \multicolumn{2}{|l|}{50,885} & \multicolumn{4}{|l|}{1829} \\
\hline & \multicolumn{2}{|c|}{ All putative SNPs(MAF > 0.05) $=95,234^{*}$} & \multicolumn{4}{|c|}{ Total No. of SNPs with allelic imbalance $=7930^{* *}$} \\
\hline
\end{tabular}

Allelic imbalances were calculated at $>2$ for amplification and $<0.5$ for loss of heterozygosity. SNPs explicitly existing in only the high or low phenotypic group are indicated in the table by the $0.0 / 1.0$ ratio. ${ }^{*} 59$ SNPs were multi-allelic, showing different alleles in association with different phenotypes. ${ }^{*} 1$ SNP was multi-allelic showing different alleles predicted by different pipelines

allelic imbalances. Out of the 7930 SNPs with allelic imbalances, there were 6038 SNPs with available read count for both alleles in the divergent families. Alternatively, there were 1892 with counts for only one allele. These SNPs existed in families at one of the two ends of each trait variation scale but not in the other. We performed chi-square test on the 6038 SNPs and found 5330 SNPs (83\%) with P_value $<0.05$ and 710 SNPs (17\%) with $P_{-}$value greater $>0.05$ (Additional file 1).

For subsequent analyses, we combined SNPs from GATK and SAMtools/Popoolation2 into three different groups: 1) Non-redundant SNPs with allelic imbalances from both methods (7930 SNPs); 2) Common putative SNPs from both methods (50,885 SNPs); 3) Putative non-redundant SNPs from both methods (95,234 SNPs) (Table 1). All SNPs data are provided in Additional file 1.

\section{SNP validation}

A total of 92 putative SNPs including 88 SNPs from the GATK/SAMtools common pool (50,885 SNPs) were selected for SNP validation. Among the 92 putative SNPs, 68 SNPs showed allelic imbalances (Table 2), including 25 SNPs identified by GATK pipeline, 10 SNPs identified by SAMtools pipeline, and 33 SNPs identified by both pipelines (Table 2). Among the 92 tested SNPs, 72 (78.2\%) SNPs were polymorphic, 11(11.9\%) SNPs were monomorphic and 9 failed the assay (Table 2). Failure of the Fluidigm assay can be caused by unsuccessful or non-specific primer binding to the target genomic DNA. Therefore, we cannot assume that a failed assay indicates failure of our bioinformatics pipeline to detect a SNP in the RNA sequence data, and can remove the failed SNP assays from the calculation of SNP validation rate. As 72 out of the 83 working Fluidigm SNP assays were polymorphic, we can claim $86.7 \%$ validation rate in detecting polymorphic SNPs in the overall putative SNP pool and 90\% validation rate in the GATK/SAMtools shared SNPs pool. This success rate is much higher than what we previously achieved in rainbow trout using RNA-Seq (70\%) and genomic reduced representation libraries $(48 \%)[11,33]$. The improved success rate in this study is perhaps due to use of a reference genome instead of de novo assembled references used in the previous studies. In addition, a transcriptome sequence coverage of $\sim 7.4 \mathrm{X}$ per fish was used compared to only $\sim 0.97 \mathrm{X}$ in our previous RNA-Seq study [11]. The 90\% successful SNP validation rate is comparable to that reported in diploid fish or using genomic RADs and doubled haploid fish in rainbow trout [7, 34]. In addition, a recent rainbow trout genome re-sequencing study with at least $10 \times$ genome coverage per fish had $86 \%$ successful validation rate [7]. Relatively lower success rates in SNP detection were

Table 2 Number of putative and validated SNPs from each dataset

\begin{tabular}{|c|c|c|c|c|c|}
\hline$\underline{\text { SNP Group }}$ & Total SNPs & Polymorphic & Monomorphic & Failed assay & Success rate \\
\hline All putative SNPs $(95,234)$ & 92 & 72 & 11 & 9 & $86.7 \%$ \\
\hline GATK/SAMTool common SNPs $(50,289)$ & 88 & 72 & 8 & 8 & $90.0 \%$ \\
\hline Total SNPs with allelic imbalance & 68 & 55 & 7 & 6 & $88.7 \%$ \\
\hline GATK unique SNPs with allelic imbalance & 25 & 21 & 3 & 1 & $87.5 \%$ \\
\hline SAMTool unique SNPs with allelic imbalance & 10 & 4 & 2 & 4 & $66.7 \%$ \\
\hline GATK/SAMTool common SNPs with allelic imbalance & 33 & 30 & 2 & 1 & $93.8 \%$ \\
\hline
\end{tabular}


reported from RNA-Seq studies in rainbow trout due to genome duplication and assembly errors in the genome/ transcriptome references $[11,35,36]$. Noteworthy and in a separate study, we found variation in gene expression in only 75 genes distributed between all 5 traits (data will be published elsewhere). Therefore, differential gene expression effects on estimating allelic imbalances were negligible as only 75 genes distributed between all five traits were differentially expressed between the high and low families. Minor effects of variation in gene expression on allele frequency estimation accuracy were previously reported [37]. The SNP validation data, albeit small, indicated that the GATK method was more successful in calling polymorphic SNPs with allelic imbalances than the SAMtools pipeline; $87.5 \%$ versus $66.7 \%$, respectively. However, combined GATK and SAMtools data had a 93.8\% success rate. Success rates between SNPs with and without allelic imbalances were $88.7 \%$ and $86.7 \%$, respectively. Importantly and out of 72 validated SNPs, 61 (84.7\%) and 58 SNPs (80.5\%) were polymorphic in fish from two different commercially important rainbow trout populations in the US, Troutlodge Inc. and Clear Springs Foods Inc., respectively. These results suggest that the SNPs identified in this study are also useful for other commercial rainbow trout populations.

To evaluate ability of the pipeline in calculating allelic imbalances, DNA and cDNA of the 35 fish used for RNA-Seq analyses of high versus low muscle yield were also genotyped. For all 72 validated SNPs, all DNA and cDNA genotypes were consistent except for $4.64 \%$ that indicated mono-allele specific gene expression as explained below.

\section{Assessment of mono-allelic gene expression}

Out of the 72 validated polymorphic SNPs (Table 2), there were 46 SNPs that showed potential mono-allelic expression in cDNA in at least one fish. In other words, the genomic DNA is heterozygous for the SNP while cDNA is monomorphic. Thirty-three of the 35 fish showed mono-allelic expression in at least one SNP. Out of the aforementioned 46 SNPs, 5 SNPs were randomly selected for validation using Sanger sequencing. All SNPs were heterozygous at the DNA level. However, manual investigation of the cDNA sequence chromatograms exhibited existence of substantial allelic imbalances ranging from existence of two alleles with $>2.0 \mathrm{X}$ peak height ratios between the 2 alleles at the SNP base to a complete mono-allelic expression (a single peak). Overall, approximately $4.64 \%$ random mono-allelic/allelic imbalances existed in gene expression of rainbow trout. These data are consistent with a recent study in human stem cells showing that most allelic imbalances did not represent 'on/off' events, but instead revealed biased expression from each allele [38]. None of the 8 tested families in our study showed mono-allelic expression in all individuals specific to a given family, indicating no parental origin effect through genomic imprinting. Likewise, the human stem cell study suggested that most of the allele-biased gene expression is not due to genomic imprinting [38]. Compared to our estimated 4.64\% mono-allelic expression, recent studies showed $12-24 \%$ random mono-allelic expression in mammals and 7-9\% in interspecies catfish [4, 39-41]. Our mono-allelic expression assessment is based on only 72 SNPs, and hence a genome-wide assessment of mono-allelic expression in rainbow trout warrants further investigation.

\section{SNP genomic/functional classification}

Three sets of SNPs were considered for genomic/functional classifications. For the 7930 SNPs with allelic imbalances, 2898 (37.69\%) were intergenic. Of them, 635 (8.01\%) and 721 (9.09\%) SNPs were located within $5 \mathrm{~Kb}$ upstream or downstream of protein-coding genes, respectively. The rest of the intergenic SNPs, 1633 (20.59\%) were located more than $5 \mathrm{~Kb}$ distant to proteincoding genes.

On the other hand, 4941 (62.31\%) SNPs were genic, including $214(2.70 \%)$ that were located within the $5^{\prime}$ untranslated region (5'UTR) and 1677 (21.15\%) that were located in the $3^{\prime}$ untranslated region (3'UTR) of protein coding genes. In addition, 2548 (32.13\%) SNPs were located within coding DNA sequences (CDS) and 502 (6.33\%) SNPs were located within introns. Of the CDS SNPs, 504 (6.36\%) were non-synonymous; 4 of these caused early stop codon, and 500 caused amino acid substitution (Table 3). There were 684 (8.63\%) SNPs located within 295 lncRNAs (Table 3).

Regarding the GATK/SAMtools shared SNPs $(50,885$ SNPs), there were 20,356 (40.00\%) intergenic SNPs. Of these shared SNPs, 4594 (9.03\%) were located within $5 \mathrm{~Kb}$ upstream, and 5208 (10.23\%) downstream of protein-coding genes. In addition, 10,554 (20.74\%) were intergenic, more than $5 \mathrm{~Kb}$ distant to protein-coding genes. In contrast, $30,529(60.00 \%)$ SNPs were genic. And, $1389(2.73 \%)$ of these SNPs were in the 5'UTR; $10,259(20.16 \%)$ were in the 3'UTR, 15,178 (29.83\%) were within CDS; and 3703 (7.28\%) were within introns. Out of those within CDS SNPs, 3919 (7.70\%) were non-synonymous SNPs. Fifty of these CDS SNPs were nonsense (causing premature stop codon), and 3869 (7.60\%) were missense SNPs (Table 3).

Concerning all the putative SNPs, there were 46,901 (49.25\%) intergenic SNPs. Of these, 9005 (9.46\%) were located within $5 \mathrm{~Kb}$ upstream; and 10,245 (10.76\%) were downstream of protein-coding genes. In addition, 27,651 (29.03\%) were more than $5 \mathrm{~Kb}$ distant from protein- 
Table 3 Summary of SNPs classification for different SNP sets

\begin{tabular}{|c|c|c|c|c|c|c|}
\hline Functional Class & $\begin{array}{l}\text { SNPs with allelic } \\
\text { imbalance } 7.9 \mathrm{~K}\end{array}$ & $\%$ & $\begin{array}{l}\text { GATK/SAMtools } \\
\text { Common SNPs } 50.8 \mathrm{~K}\end{array}$ & $\%$ & $\begin{array}{l}\text { All putative } \\
\text { SNPs } 95.2 \mathrm{~K}\end{array}$ & $\%$ \\
\hline Intergenic & 2989 & $37.69 \%$ & 20,356 & $40.00 \%$ & 46,901 & $49.25 \%$ \\
\hline Intergenic(>5 K) & 1633 & $20.59 \%$ & 10,554 & $20.74 \%$ & 27,651 & $29.03 \%$ \\
\hline Upstream (<5 K) & 635 & $8.01 \%$ & 4594 & $9.03 \%$ & 9005 & $9.46 \%$ \\
\hline Downstream (<5 K) & 721 & $9.09 \%$ & 5208 & $10.23 \%$ & 10,245 & $10.76 \%$ \\
\hline Genic & 4941 & $62.31 \%$ & 30,529 & $60.00 \%$ & 48,333 & $50.75 \%$ \\
\hline $5^{\prime} \cup T R$ & 214 & $2.70 \%$ & 1389 & $2.73 \%$ & 2247 & $2.36 \%$ \\
\hline $3^{\prime} U T R$ & 1677 & $21.15 \%$ & 10,259 & $20.16 \%$ & 16,420 & $17.24 \%$ \\
\hline CDS & 2548 & $32.13 \%$ & 15,178 & $29.83 \%$ & 22,616 & $23.75 \%$ \\
\hline Intronic & 502 & $6.33 \%$ & 3703 & $7.28 \%$ & 7050 & $7.40 \%$ \\
\hline Non-synonymous & 504 & $6.36 \%$ & 3919 & $7.70 \%$ & 5853 & $6.15 \%$ \\
\hline Stop gain & 4 & $0.05 \%$ & 50 & $0.10 \%$ & 79 & $0.08 \%$ \\
\hline Missense & 500 & $6.31 \%$ & 3869 & $7.60 \%$ & 5774 & $6.06 \%$ \\
\hline LncRNA & 684 & $8.63 \%$ & 4386 & $8.62 \%$ & 10,465 & $10.99 \%$ \\
\hline Total number/percentage & 7930 & $100.00 \%$ & 50,885 & $100.00 \%$ & 95,234 & $100.00 \%$ \\
\hline
\end{tabular}

coding genes. Alternatively, 48,333 (50.75\%) SNPs were genic, and of these genic SNPs, 2247 (2.36\%) were in the 5'UTR; 16,420 (17.24\%) were in the 3'UTR; 22,616 (23.75\%) were within CDS; and 7050 (7.40\%) were within introns. Of the CDS SNPs, 5853 (6.15\%) were nonsynonymous with 79 SNPs causing early stop codons and 5774 (6.06\%) causing amino acid changes (Table 3 ).

In these three SNP datasets, there were large percentages of intergenic (including upstream/downstream) SNPs (37-49\%). Approximately $10 \%$ intergenic in addition to $30 \%$ non-coding SNPs were reported in humans from RNA-Seq data [42]. Our high percentages of intergenic SNPs may be partially explained by the incomplete annotation of protein coding genes and exons in the current version of the rainbow trout reference genome sequence [28].

\section{Distribution and density of SNPs in the genome}

Chromosome density distribution of the SNPs with allelic imbalances exhibited high density for all five traits in several chromosomes with the three highest peaks in chromosomes 9, 20 and 28 (Fig. 2a). All five traits revealed very similar pattern of distribution with a single exception; shear force exhibited a relative higher density than the other traits on chromosome 9. The similarity in density distribution between traits may be explained at least in part by the positive correlation that we observed between the phenotypes in this population. WBW and thermal growth coefficient were used as selection criterion in this population $[11,26]$, and we found that WBW as an independent variable has significant effects on muscle yield and fat percentage (multivariable regression analysis $[P<0.01]$, data not shown). However, despite the similarity in SNP density distributions, most of the identified SNPs were unique to each trait. From the 7930 SNPs with allelic imbalances, only 27 were shared by all five traits, 161 were shared by four traits, 680 were shared by three traits and 1783 were shared by two traits. In agreement with our results, a recent GWAS study identified two windows with effect on fillet yield located on chromosome 9 and explaining 1.0-1.5\% of genetic variance in the same fish population [6].

As can be expected, the number of SNPs with allelic imbalances per chromosome was strongly correlated with chromosome length (Fig. 2b). In general, numbered unknown chromosomes, which are longer in the current reference genome [28], had more SNPs compared to the known chromosomes (Fig. 2b). Chromosome "Unknown" (1.1 Gb of scaffolds not assigned to chromosomes) had 4086 (49.05\%) SNPs (not shown in Fig. 2b). Previous genetic mapping reports showed that the growth-related SNPs/QTL are distributed over $\sim 20$ chromosomes $[11,43,44]$. Together with our data, these reports confirm the polygenetic nature of growth/muscle related traits in rainbow trout.

\section{SNP functional annotation}

Functional annotation of genes harboring SNPs with allelic imbalances were performed using the Blast2GO suite [45]. The SNP-flanking sequences were searched against the NCBI nr-protein database using BLASTx; then, associated genes and Gene Ontology (GO) terms were acquired. In the biological processes category, SNP-harboring genes were associated with various cellular processes mainly involved in growth-related mechanisms, including regulation of metabolic and oxidation- 




Fig. 2 Genome distribution of the SNPs with allelic imbalances for all five traits. SNP density (SNPs per 100,000 NT) (a) and total number of SNPs (b) are shown for each chromosome. Chromosome "Unknown" (1.1 Gb scaffolds not assigned to chromosomes) had 4086 (49.05\%) SNPs is not shown in the lower panel

reduction processes and protein translation (Fig. 3). In the molecular function category, SNP-containing genes were associated with binding metal ions, ATP, nucleic acid, and actin. In addition, a significant number of the genes were associated with transferase, motor, oxidoreductase, and structural molecule activities (Fig. 3). In the cellular component category, many of the genes exhibited association with the cytoplasmic compartment, membranes, myosin complex, and extracellular region compartment (Fig. 3). Genes with similar GO associated terms were previously reported to be involved in rainbow trout muscle growth and quality [11, 19, 43, 46-48].

Additionally, KEGG pathway mapping was used to assign enzyme function to the SNP-containing transcripts [49]. Searching transcripts against the KEGG database yielded 1043 transcripts (13.15\%) with significant KEGG hits to 632 KEGG Orthologies (KOs) belonging to different pathways (Table 4). Most of the transcripts were assigned to growth-related metabolic pathways. There were 275 transcripts $(182 \mathrm{KOs})$ related to metabolism.
Under this category, sequences matching energy metabolism (88 transcripts, $57 \mathrm{KOs}$ ) appeared on the top of the list, with 52 transcripts ( $37 \mathrm{KOs}$ ) assigned to oxidative phosphorylation. Sequences matching carbohydrate metabolism occupied the second place (77 transcripts, $43 \mathrm{KOs}$ ) and were further classified into glycolysis/gluconeogenesis (39 transcripts, $18 \mathrm{KOs}$ ), citrate cycle (19 transcripts, $14 \mathrm{KOs})$ and pyruvate metabolism (16 transcripts, 10 enzymes). The next metabolic subcategories in the metabolic list were amino acid metabolism (56 transcripts, $41 \mathrm{KOs}$ ), lipid metabolism (27 transcripts, 22 $\mathrm{KOs}$ ), and cofactors and vitamins metabolism (14 transcripts, $11 \mathrm{KOs}$ ). These preliminary SNP functional annotations are in agreement with previous reports that showed strong association between 1) mutations and altered expression of glycolytic and oxidative phosphorylation enzymes and 2) rainbow trout growth and muscle degeneration [11, 19, 43, 46, 47].

In addition, 176 KEGG annotated sequences were assigned to the genetic information processing category 


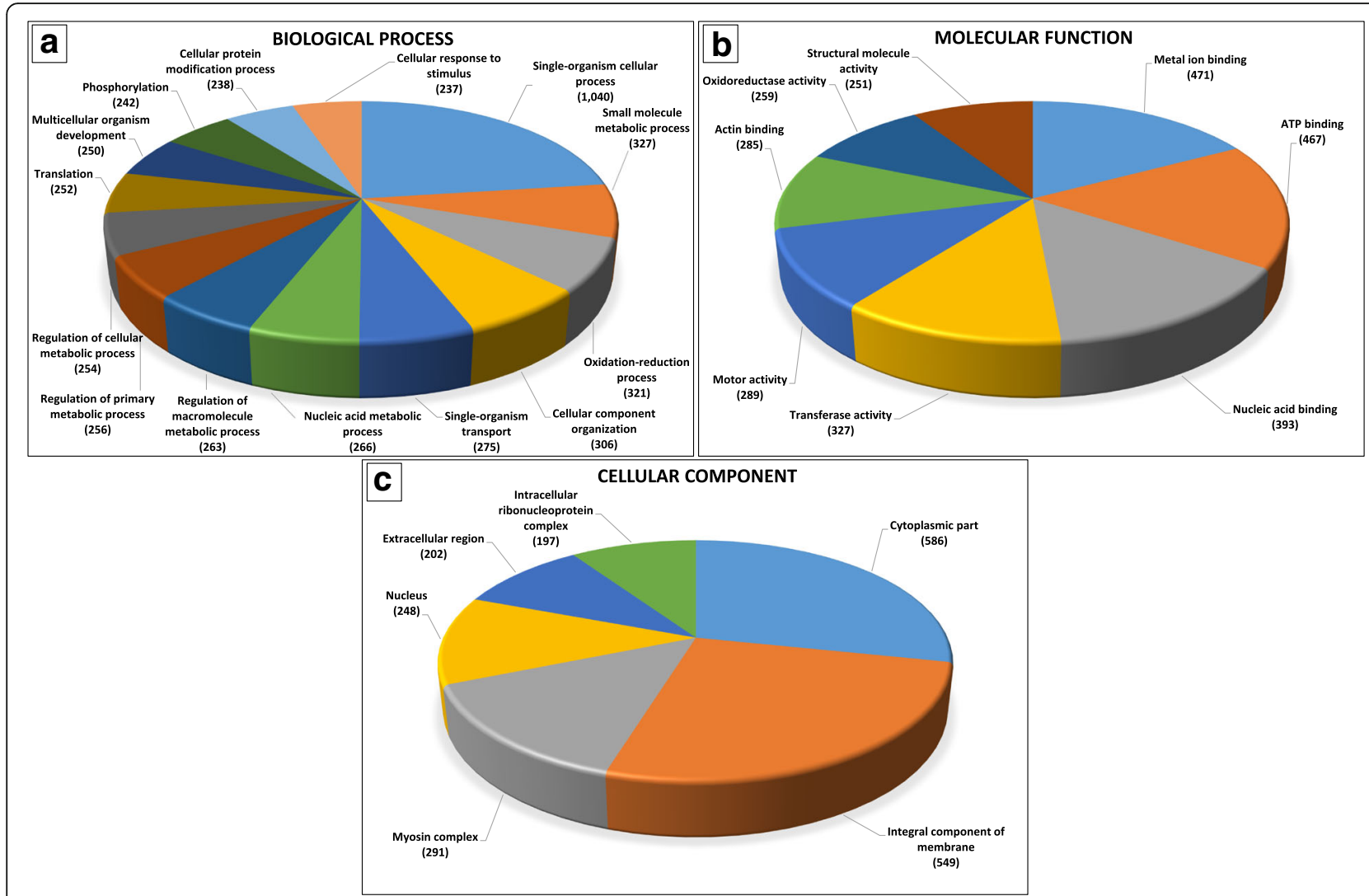

Fig. 3 Gene Ontology (GO) assignment of the genes harboring SNPs with allelic imbalances in families with contrasting growth and muscle phenotypes. Genes were assigned GO terms according to their biological processes (a), molecular functions (b) and cellular components (c)

(112 KOs) that included translation (105 sequences, 69 KOs), folding, sorting and degradation (62 sequences, 38 KOs), and transcription (9 sequences, $5 \mathrm{KOs}$ ) (Table 4). A significant number of the SNP-harboring genes matched ribosomal (68 sequences, $48 \mathrm{KOs}$ ) and RNAtransport proteins (22 sequences, $12 \mathrm{KOs}$ ). Previously, we showed that the atrophying muscle and muscle from fast versus slow growing rainbow trout had differentially expressed genes involved in RNA processing, protein synthesis, posttranslational modification, and intracellular protein trafficking $[19,43,46]$.

Moreover, 166 sequences (99 KOs) were classified by KEGG mapping into the environmental information processing category; these sequences were further assigned to signal transduction (147 sequences, $87 \mathrm{KOs}$ ) and signaling and interaction molecules (19 sequences, $12 \mathrm{KOs}$ ) (Table 4). The PI3K-Akt signaling, Calcium signaling, MAPK signaling, and cGMP-PKG signaling pathways had the largest numbers of hits: $21,18,18$, and $16 \mathrm{KOs}$, respectively. Previous studies indicated involvement of MAPK and Calcium signaling in fish/muscle growth [46, 50].

Furthermore, the cellular processes category contained 152 KEGG-annotated sequences matching 85 KOs, which were further classified into cellular community
(54 transcripts, $27 \mathrm{KOs}$ ), transport and catabolism (42 transcripts, $24 \mathrm{KOs}$ ), and cell growth and death (36 transcripts, 22 KOs) (Table 4). In the organismal systems category, the most significant subcategories were endocrine (105 transcripts, $53 \mathrm{KOs}$ ), circulatory (49 transcripts, $30 \mathrm{KOs}$ ), immune (44 transcripts, $28 \mathrm{KOs}$ ), and digestive systems (32 transcripts, 16 KOs). Recently, a GWAS study using the same fish population identified a small number of genes involved in muscle development explaining $\sim 1.0 \%$ of the total genetic variance of the muscle yield and growth rate [6].

Distributions of KEGG matches were generally similar among all five traits. Albeit, we noticed an increased number of hits related to fillet whiteness compared to other traits, for carbohydrate metabolism (47 transcripts, $28 \mathrm{KOs}$ ) and amino acid metabolism (32 transcripts, 26 KOs) (Table 4). Similarly, there was a noticeable increase in numbers of hits in whiteness for PI3K-Akt signaling, focal adhesion, gap junction and regulation of actin cytoskeleton (Table 4). Regulation of focal adhesion and actin cytoskeleton were associated with development of pale, soft, and exudative (PSE) meat in turkey [51]. In addition, the muscle yield trait exhibited an increased number of transcripts for energy metabolism, with 28 


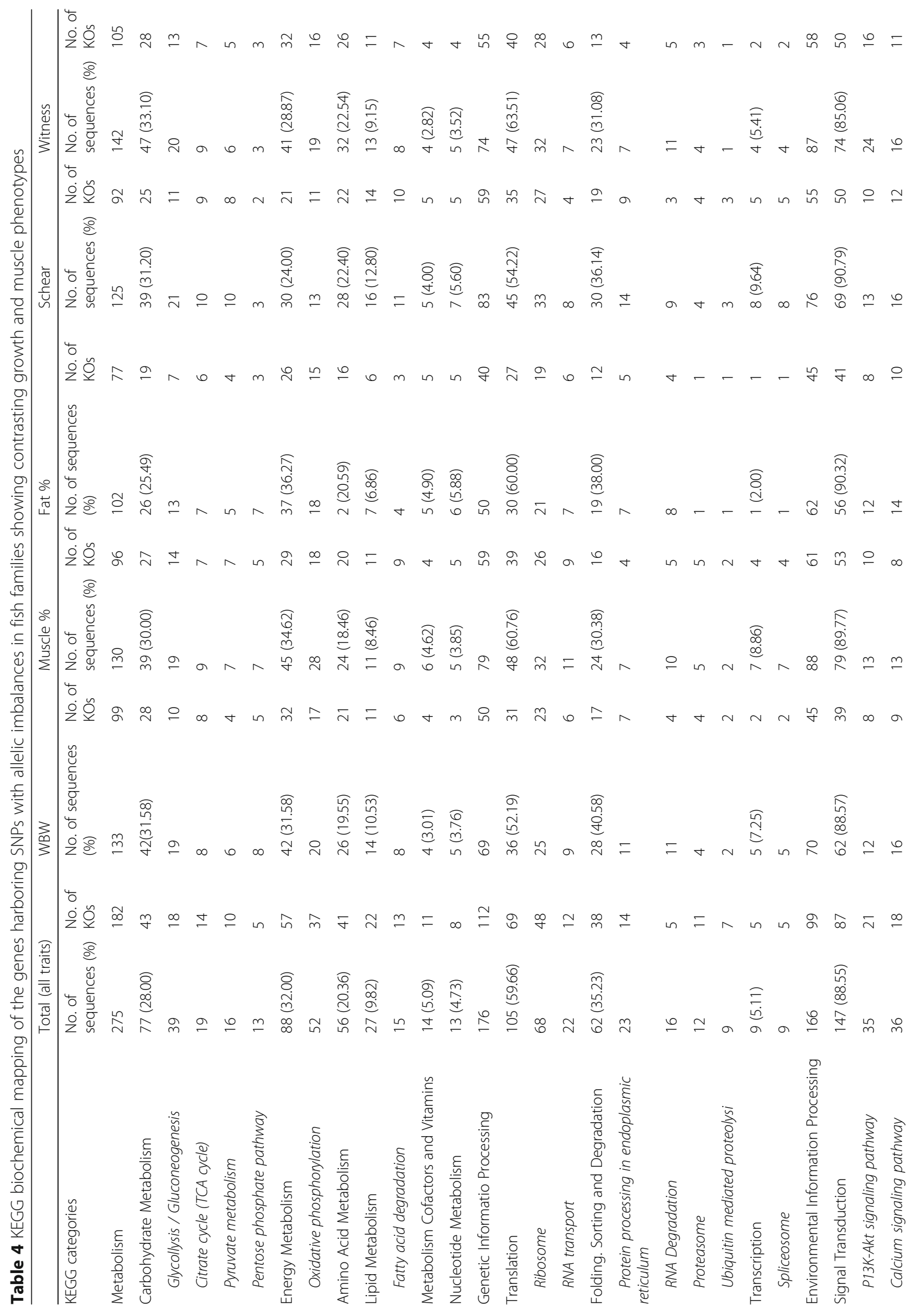




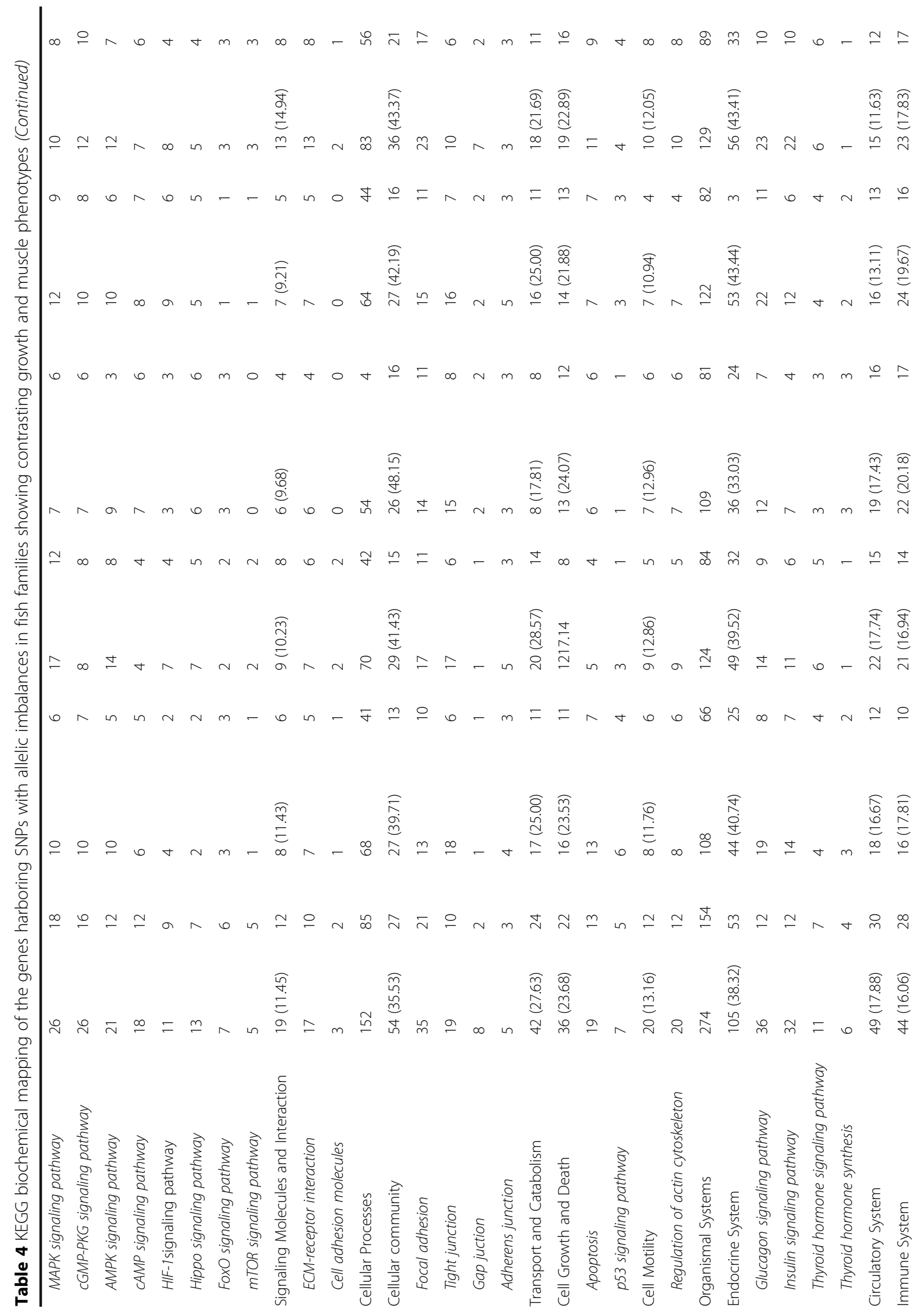




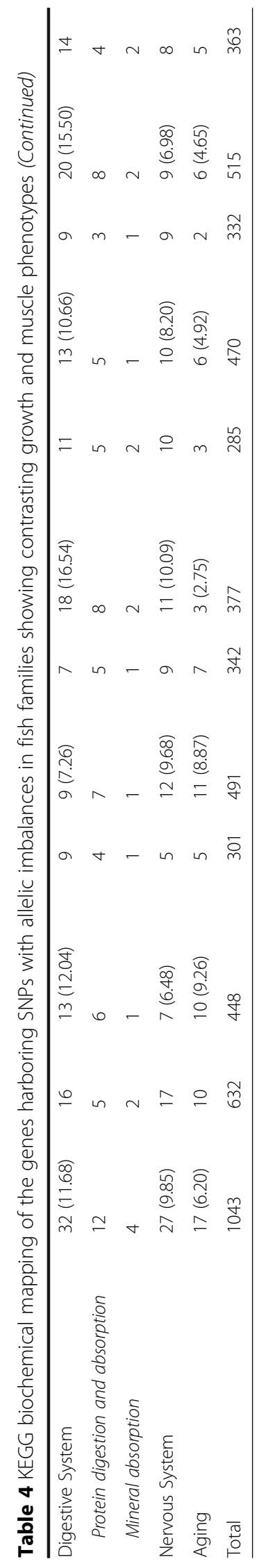


transcripts/18 KOs belonging to oxidative phosphorylation. Shear force exhibited an increased number of transcripts belonging to lipid metabolism (16 transcripts, 14 KOs) (Table 4).

Our KEGG pathway mapping results have linked many of the genes harboring SNPs with allelic imbalances to potential regulation of growth and metabolic pathways, which may support pathway-based GWAS analyses in rainbow trout, similar to what has been recently applied to detect genetic pathways explaining live weight and muscle growth variation in cattle genotypes [52].

\section{Methods}

\section{Fish population, sampling and sequencing}

Phenotypic data and muscle samples were collected from $\sim 500$ fish representing 98 families (5 fish/family) from the growth-selected line at NCCCWA (year class 2010) as previously described $[6,11,26]$. Families were produced and reared until $\sim 13$ months post-hatch as described in reference [26]. Briefly, full-sib families were produced from single-sire $\times$ single-dam matings. Eggs were reared in spring water, and water temperatures were manipulated between approximately 7 and $13{ }^{\circ} \mathrm{C}$ to synchronize hatch times. Each family was stocked separately in 200-L tanks at a density of approximately $600 \mathrm{al}$ evins/tank. Fish were randomly culled every month to maintain stocking densities $<50 \mathrm{~kg} / \mathrm{m} 3$. At about 5months old, fish were anesthetized using $100 \mathrm{mg} / \mathrm{L}$ of tricaine methanesulfonate (Tricaine-S, Western Chemical, Ferndale, WA) and uniquely tagged by inserting a passive integrated transponder (Avid Identification Systems Inc., Norco, CA) into the dorsal musculature, and tagged fish were combined and reared in 1000-L communal tanks. Fish were fed a commercial fishmealbased diet (42\% protein, 16\% fat; Ziegler Bros Inc., Gardners, PA) using automatic feeders (Arvotec, Huutokoski, Finland). Initially, young fish were fed at a daily feeding rate $\sim 2.5 \%$ of body weight (BW), which later was gradually reduced to approximately $0.75 \%$ of BW.

Fish were sampled as previously described for year class 2010 in Gonzalez-Pena et al., publication [6]. Briefly, WBW was measure in fish belonging to 98 families and families were sorted according to their WBW. The 2nd or 3rd fish from each family was selected for muscle sampling to keep the distribution of WBW consistently adjusted around the median of each family. Selected fish were randomly assigned to one of five harvest groups ( 100 fish each) allowing one fish per family per harvest group. The five groups were sampled in five consecutive weeks (one group/week). Fish were samples at about $~ 13$-months old (410-437 days post-hatch, mean body weight $=985 \mathrm{~g} ; \mathrm{SD}=239 \mathrm{~g})$. At harvest, fish were anesthetized in approximately $100 \mathrm{mg} / \mathrm{L}$ of tricaine methane sulfonate (Tricaine-S, Western Chemical, Ferndale, WA).

At harvest, a muscle sample was excised from the left dorsal musculature and frozen in liquid nitrogen for subsequent RNA sequencing. Fish were slaughtered, and eviscerated then head-on gutted carcasses were packed in ice, transported to the West Virginia University Meats Processing Laboratory (Morgantown, WV), and stored overnight. The next day, carcasses were hand-processed into trimmed, skinless fillets by a trained faculty member and weighed. Muscle yield and quality analyses were conducted as previously described [53]. Briefly, muscle yield was calculated as a percent of muscle weight relative to WBW. A $40 \times 80 \mathrm{~mm}$ muscle section was separated, parallel to the long axis of the body, from the dorsal musculature for texture analysis [54]. The remaining muscle from the fillets was pulverized with liquid nitrogen in a Waring Blender (Waring, New Hartford, CT) and kept at $-25{ }^{\circ} \mathrm{C}$ for chemical composition analyses. Proximate composition of muscle was determined using AOAC [55] approved methods. Crude fat was analyzed using the Soxhlet solvent extractor with petroleum ether. Texture of fillet sections was determined using a five-blade, Allo-Kramer shear cell attached to a Texture Analyzer (Model TA-HDi ${ }^{\circ}$; Texture Technologies Corp., Scarsdale, NY), equipped with a 50$\mathrm{kg}$ load cell and at a crosshead speed of $127 \mathrm{~mm} / \mathrm{min}$. Force-deformation graphs were recorded and analyzed using the Texture Expert Exceed software (version 2.60; Stable Micro Systems Ltd., Surrey, U.K.). Peak shear force (g/g sample) was recorded.

Fresh fillet surface color was measured with a Chroma meter (Minolta, Model CR-300; Minolta Camera Co., Osaka, Japan) calibrated using a standard white plate No. 21333180 (CIE Y 93.1; × 0.3161; y 0.3326). L* (lightness), $a^{*}$ (redness), and $b^{*}$ (yellowness) values were recorded at three locations above the lateral line along the long axis of the right fillet, and these values were used to calculate a fillet whiteness index according to the following equation: Whiteness $=100-\left[(100-\mathrm{L})^{2}+\mathrm{a}^{2}+\mathrm{b}^{2}\right]^{1 / 2}[81]$.

For RNA-Seq analyses, out of 98 families measured for phenotypic data, eight families (5 fish each) showing opposite phenotypes for each of the 5 traits were analyzed (4 high ranked families versus 4 low ranked families on average for each trait). Since some fish families were common between the traits, the total number of selected families for RNA-Seq was 22 families. Total RNA was isolated from each fish muscle sample using TRIzol ${ }^{\mathrm{mm}}$ (Invitrogen, Carlsbad, CA). Equal masses of total RNA from 5 samples of each family were pooled and used for RNA-Seq sequencing. cDNA libraries were prepared and sequenced on an Illumina HiSeq (single-end, 100 bp read length) using multiplexing standard protocols as previously described [56]. Briefly, mRNA was selected 
from one microgram of high quality total RNA. Firststrand synthesis was synthesized with a random hexamer and SuperScript II (Life Technologies). Double stranded DNA was blunt-ended, 3'-end A-tailed and ligated to indexed adaptors. The adaptor-ligated double-stranded cDNA was amplified by PCR for 10 cycles with the Kapa HiFi polymerase (Kapa Biosystems, Woburn, MA) to reduce the likeliness of multiple identical reads due to preferential amplification. The final libraries were quantitated Qubit (Life Technologies, Grand Island, NY) and the average size was determined on an Agilent bioanalyzer DNA7500 DNA chip (Agilent Technologies, Wilmington, $\mathrm{DE}$ ), diluted to $10 \mathrm{nM}$ and the indexed libraries were pooled in equimolar concentration before sequencing.

\section{SNP detections using SAMtools/Popoolation2}

For each trait (WBW, muscle yield, muscle fat content, shear force, and whiteness), sequence reads from each family were aligned to the rainbow trout genome using STAR [29]. After read alignment, the SAMtools view/ sort and mpileup functions were used within the Popoolation2 package (version 1.201) to determine the genotype for each variant and calculate allele frequencies [57, 58]. Initial SNPs were considered at minimum reads $>10$ and minor allele count $>4$ and MAF $>0.05$. Putative SNPs associated with each trait were determined by calculating SNP allelic imbalance scores as previously described [11, 59]. A SNP allelic imbalance score was determined by assessing the ratio of [frequency of allele $\mathrm{A} /$ frequency of allele $\mathrm{B}$ in high-end families]/[frequency of allele $\mathrm{A} /$ frequency of allele $\mathrm{B}$ in the corresponding low-end families]. The allelic imbalances score ranges from zero to infinity. SNPs with allelic imbalance were called if the ratio is more than or equal 2.0 (as an amplification) or less than or equal 0.5 (as loss of heterozygosity. The phase of the alleles could not be determined for families surveyed since the parental genotypes were not known for most of the fish. Allele counts in the divergent families were extracted from the VCF files. Chisquare test of two-by-two Tables [60] was performed with $p$-value $<0.05$ to determine if SNPs that are showing allelic imbalances are statistically significant.

\section{SNP detection using GATK tools}

For the GATK pipeline [61], reads from each sample were aligned to the rainbow trout genome using STAR [29] as recommended by the GATK practice. Picard tools were used to sort the SAM files and to mark duplicates, a step used by GATK to reduce a false positive due to error in duplicate that could be falsely detected as a SNP. The following steps were performed according to GATK pipeline for RNA-Seq (Split and trim to reassign mapping quality, Indel realignment, local realignment around Indel in order to clean up any mapping artifacts and Base Quality Score Recalibration). After data preparation, variants were called using HaplotypeCaller followed by hard-filtering using the following parameters: Qual By Depth (QD) 2.0, FisherStrand (FS) 60.0: RMS Mapping Quality (MQ) 40.0, MAF > 0.05. Since GATK was not optimized to calculate allelic imbalances in RNA-Seq data, putative SNPs identified in each family were analyzed using an in-house Perl script to determine the allelic imbalances applying the criteria that we used in the SAMtools/Popoolation2 method.

\section{SNP validation}

Flanking sequences (up to $250 \mathrm{bp}$ on each side) of putative SNPs were extracted from the reference genome [28]. Some SNPs were removed from SNP assay design because either a sequence gap was located less than $60 \mathrm{bp}$ from the SNP site or a non-target SNP was located less than 30 bp away from the target SNP. A total of 92 SNP assays were developed and evaluated with 282 DNA or cDNA samples. These included 85 DNA samples derived from 19 full-sib families used for RNA-Seq and their parents (38 DNA samples), DNA samples of 2 full-sib mapping families (2 parents and 19 offspring per family), 64 DNA samples from two commercial populations (Troutlodge Inc. and Clear Springs Foods Inc.) and 35 cDNA samples derived from the RNA samples used for RNA-Seq high versus low muscle yield. The SNP genotyping was performed following the instructions of the Fluidigm genotyping user guide. Briefly, DNA and cDNA samples were pre-amplified, diluted and used for genotyping with 96.96 Dynamic Array IFCs (Integrated Fluidic Circuits). The arrays were read using EP1 system, and genotypes were called automatically using Fluidigm SNP genotyping analysis software 4.1 with a confidence threshold of 85 . The genotype clusters were examined for each assay and any wrong calls or no calls were corrected manually. The program Pedcheck [62] was used to identify genotypes inconsistent with Mendelian inheritance between parents and offspring. Chi-square goodness of fit tests were performed to identify SNPs with significant segregation distortion $(P<0.01)$ in the two mapping families. Those SNPs were reported as assay-failed SNPs.

For the Sanger sequencing validation of the SNPs showing potential mon-allelic gene expression, flanking sequences (up to $250 \mathrm{bp}$ on each side) of each SNP were PCR amplified from DNAs and cDNA from the same 35 fish samples that were used for RNA-Seq high versus low muscle analyses. PCR amplicons were Sanger sequenced and manually inspected for consistency between DNA and cDNA genotypes or mono-allele specific gene expression as explained in the results section. 


\section{Functional annotation of SNPs}

SNP annotation by functional class (genic/intergenic etc.) for different SNP sets and their genome distributions were conducted using in-house Perl scripts. The gff file of the rainbow trout genome reference [28] was used to determine if a SNP is located within an mRNA start and end positions (genic), within a CDS, 5'UTR or 3'UTR. SNPs not within start and end positions of mRNA were considered intergenic. Upstream/ downstream intergenic SNPs were determined if located within $5 \mathrm{~kb}$ of an mRNA. SNPs within lncRNAs were determined using gtf file of our previously reported lncRNA reference [63]. Functional annotation of the SNP-harboring genes was performed using the Blast2GO suite [30] and KEGG pathway mapping.

\section{Additional file}

Additional file 1: Putative SNPs and SNPs with allelic imbalances in association with total body weight, muscle yield, muscle fat content, shear force, and whiteness. SNP chromosome position, alleles, functional classification, associated gene ID and FASTA sequences are provided. (CSV $15332 \mathrm{~kb}$ )

\section{Abbreviations}

CDS: Coding DNA sequences; FS: Fisher Strand; GATK: Genome Analysis Toolkit v3.3.0; GO: Gene Ontology; KOs: KEGG Orthologies; MAF: Minor allele frequency; MQ: Mapping Quality; PSE: Pale, soft, and exudative; QD: Qual By Depth; WBW: Whole body weight

\section{Acknowledgments}

Not applicable.

\section{Funding}

This study was supported by a competitive grant No. 2014-67,015-21,602 from the United States Department of Agriculture, National Institute of Food and Agriculture (MS)

\section{Availability of data and materials}

All SNP data are provided in additional file 1 and were deposited in the NCBI dbSNP database (accession number from ss\#2711191806 to 2,711,287,038 in addition to ss\# 2137497773). While the manuscript was under review for publication, a newer assembly of the rainbow trout genome was deposited at NCBI (GenBank: MSJN00000000.1, Dr. Palti personal communication). SNP flanking sequences were mapped to the new reference and positions of the uniquely mapped SNP sequences were annotated in Additional file 1.

\section{Authors' contributions}

Conceived and designed the experiments: MS, TL, BK. Performed the experiments: RA, MS, TL, BK. Analyzed the data: RA, AA, SL, YP, BK, MS. Wrote the paper: MS, RA, AA. All authors reviewed and approved the publication.

\section{Ethics}

Institutional Animal Care and Use Committee of the United States Department of Agriculture, National Center for Cool and Cold Water Aquaculture (Leetown, WV specifically reviewed and approved all husbandry practices used in this study (IACUC approval \#056).

\section{Consent for publication}

Not applicable.

\section{Competing interests}

The authors declare that they have no competing interests.

\section{Publisher's Note}

Springer Nature remains neutral with regard to jurisdictional claims in published maps and institutional affiliations.

\section{Author details}

${ }^{1}$ Computational Science Program, Middle Tennessee State University, Murfreesboro, TN 37132, USA. ²Department of Biology and Molecular Biosciences Program, Middle Tennessee State University, Murfreesboro, TN 37132, USA. ${ }^{3}$ National Center for Cool and Cold Water Aquaculture, ARS-USDA, Kearneysville, WV 25430, USA. ${ }^{4}$ Division of Animal and Nutritional Sciences, West Virginia University, Morgantown, WV 26506, USA.

Received: 26 December 2016 Accepted: 1 August 2017 Published online: 07 August 2017

\section{References}

1. Salem M, Kenney PB, Rexroad CE, Yao J. Molecular characterization of muscle atrophy and proteolysis associated with spawning in rainbow trout. Comp Biochem Physiol Part D Genomics Proteomics. 2006;1(2):227-37.

2. Gjedrem T. Selection and breeding programs in aquaculture. New York: Springer; 2008.

3. Rexroad CE, Palti Y, Gahr SA, Vallejo RL. A second generation genetic map for rainbow trout (Oncorhynchus Mykiss). BMC Genet. 2008;9:74-4.

4. Wang R, Sun L, Bao L, Zhang J, Jiang Y, Yao J, Song L, Feng J, Liu S, Liu Z. Bulk segregant RNA-seq reveals expression and positional candidate genes and allele-specific expression for disease resistance against enteric septicemia of catfish. BMC Genomics. 2013;14:929.

5. Wang S, Sha Z, Sonstegard TS, Liu H, Xu P, Somridhivej B, Peatman E, Kucuktas H, Liu Z. Quality assessment parameters for EST-derived SNPs from catfish. BMC Genomics. 2008;9:450.

6. Gonzalez-Pena D, Gao G, Baranski, M., Moen, T., , Cleveland B, Kenney P, Vallejo R, Palti Y, Leeds T: Genome-wide association study for identifying loci that affect fillet yield, carcass, and body weight traits in rainbow trout (Oncorhynchus Mykiss). . Front Genet 2016, 7.

7. Palti Y, Gao G, Liu S, Kent MP, Lien S, Miller MR, Rexroad CE, Moen T. The development and characterization of a $57 \mathrm{~K}$ single nucleotide polymorphism array for rainbow trout. Mol Ecol Resour. 2015;15(3):662-72.

8. Tsai HY, Hamilton A, Tinch AE, Guy DR, Gharbi K, Stear MJ, Matika O, Bishop SC, Houston RD. Genome wide association and genomic prediction for growth traits in juvenile farmed Atlantic salmon using a high density SNP array. BMC Genomics. 2015;16:969.

9. Carlton VE, Ireland JS, Useche F, Faham M. Functional single nucleotide polymorphism-based association studies. Hum Genomics. 2006;2(6):391-402.

10. Brookes AJ: Single Nucleotide Polymorphism (SNP). In: ENCYCLOPEDIA OF LIFE SCIENCES (e/s). Edited by John Wiley \& Sons Lwen, http://onlinelibrary. wiley.com/doi/10.1002/9780470015902.a0005006.pub2/full, accessed April 4, 2012; 2007.

11. Salem M, Vallejo RL, Leeds TD, Palti Y, Liu S, Sabbagh A, Rexroad CE, Yao J. RNA-Seq identifies SNP markers for growth traits in rainbow trout. PLoS One. 2012;7(5):e36264.

12. Villanueva B, Dekkers JC, Woolliams JA, Settar P. Maximizing genetic gain over multiple generations with quantitative trait locus selection and control of inbreeding. J Anim Sci. 2004;82(5):1305-14.

13. Dekkers JC: Commercial application of marker- and gene-assisted selection in livestock: strategies and lessons. J Anim Sci 2004, 82 E-Suppl:E313-E328.

14. Pang Y, Wang J, Zhang C, Lei C, Lan X, Yue W, Gu C, Chen D, Chen H. The polymorphisms of bovine VEGF gene and their associations with growth traits in Chinese cattle. Mol Biol Rep. 2010;

15. Tsai HY, Hamilton A, Guy DR, Tinch AE, Bishop SC, Houston RD. The genetic architecture of growth and fillet traits in farmed Atlantic salmon (Salmo Salar). BMC Genet. 2015;16:51.

16. Harvey DJ. Aquaculture Outlook. Electronic Outlook Report from the Economic Research Service wwwersusdagov: In; 2006.

17. Davidson WS. Adaptation genomics: next generation sequencing reveals a shared haplotype for rapid early development in geographically and genetically distant populations of rainbow trout. Mol Ecol. 2012;21(2):219-22.

18. Speare D, Arsenault G, Buote M. Evaluation of rainbow trout as a model for use in studies on pathogenesis of the branchial microsporidian Loma salmonae. Contemp Top Lab Anim Sci. 1998;37(2):55-8. 
19. Salem M, Kenney PB, Rexroad CE 3rd, Yao J. Microarray gene expression analysis in atrophying rainbow trout muscle: a unique nonmammalian muscle degradation model. Physiol Genomics. 2006;28(1):33-45.

20. Patel M, Rogers JT, Pane EF, Wood CM. Renal responses to acute lead waterborne exposure in the freshwater rainbow trout (Oncorhynchus Mykiss). Aquat Toxicol. 2006;80(4):362-71.

21. Thorgaard GH, Bailey GS, Williams D, Buhler DR, Kaattari SL, Ristow SS, Hansen JD, Winton JR, Bartholomew JL, Nagler JJ, et al. Status and opportunities for genomics research with rainbow trout. Comparative biochemistry and physiology Part B, Biochemistry \& molecular biology. 2002;133(4):609-46.

22. Cirulli ET, Singh A, Shianna KV, Ge D, Smith JP, Maia JM, Heinzen EL, Goedert JJ, Goldstein DB. Center for HIVAVI: screening the human exome: a comparison of whole genome and whole transcriptome sequencing. Genome Biol. 2010;11(5):R57.

23. Heap GA, Yang JH, Downes K, Healy BC, Hunt KA, Bockett N, Franke L, Dubois PC, Mein CA, Dobson RJ, et al. Genome-wide analysis of allelic expression imbalance in human primary cells by high-throughput transcriptome resequencing. Hum Mol Genet. 2010;19(1):122-34.

24. Vidal RO, do Nascimento LC, Mondego JM, Pereira GA, Carazzolle MF: Identification of SNPs in RNA-seq data of two cultivars of Glycine max (soybean) differing in drought resistance. Genet Mol Biol 2012, 35(1 (suppl)):331-334.

25. Yang SS, Tu ZJ, Cheung F, Xu WW, Lamb JF, Jung HJ, Vance CP, Gronwald JW. Using RNA-Seq for gene identification, polymorphism detection and transcript profiling in two alfalfa genotypes with divergent cell wall composition in stems. BMC Genomics. 2011;12:199.

26. Leeds TD, Vallejo RL, Weber GM, Pena DG, Silverstein JS. Response to five generations of selection for growth performance traits in rainbow trout (Oncorhynchus Mykiss). Aquaculture. 2016:465:341-51.

27. Leeds TD, Kenney PB, Manor M: Genetic parameter estimates for feed intake, body composition, and fillet quality traits in a rainbow trout population selected for improved growth. In: International Symposium on Genetics in Aquaculture 2012; Auburn, AL; 2012: 259.

28. Berthelot C, Brunet F, Chalopin D, Juanchich A, Bernard M, Noel B, Bento P, Da Silva C, Labadie K, Alberti A, et al. The rainbow trout genome provides novel insights into evolution after whole-genome duplication in vertebrates. Nat Commun. 2014;5:3657.

29. Dobin A, Davis CA, Schlesinger F, Drenkow J, Zaleski C, Jha S, Batut P, Chaisson M, Gingeras TR. STAR: ultrafast universal RNA-seq aligner. Bioinformatics. 2013;29(1):15-21.

30. McKenna A, Hanna M, Banks E, Sivachenko A, Cibulskis K, Kernytsky A, Garimella K, Altshuler D, Gabriel S, Daly M, et al. The genome analysis toolkit: a MapReduce framework for analyzing next-generation DNA sequencing data. Genome Res. 2010;20(9):1297-303.

31. Raineri E, Ferretti L, Esteve-Codina A, Nevado B, Heath S, Pérez-Enciso M. SNP calling by sequencing pooled samples. BMC Bioinformatics. 2012;13:239.

32. Li H, Handsaker B, Wysoker A, Fennell T, Ruan J, Homer N, Marth G, Abecasis G, Durbin R, Subgroup GPDP. The sequence alignment/map format and SAMtools. Bioinformatics. 2009;25(16):2078-9.

33. Sanchez CC, Smith TP, Wiedmann RT, Vallejo RL, Salem M, Yao J, Rexroad CE $3 r d$. Single nucleotide polymorphism discovery in rainbow trout by deep sequencing of a reduced representation library. BMC Genomics. 2009;10:559.

34. Palti Y, Gao G, Miller MR, Vallejo RL, Wheeler PA, Quillet E, Yao J, Thorgaard $\mathrm{GH}$, Salem M, Rexroad CE 3rd. A resource of single-nucleotide polymorphisms for rainbow trout generated by restriction-site associated DNA sequencing of doubled haploids. Mol Ecol Resour. 2014;14(3):588-96.

35. Seeb JE, Pascal CE, Grau ED, Seeb LW, Templin WD, Harkins T, Roberts SB. Transcriptome sequencing and high-resolution melt analysis advance single nucleotide polymorphism discovery in duplicated salmonids. Mol Ecol Resour. 2011;11(2):335-48.

36. Ryynanen HJ, Primmer CR. Single nucleotide polymorphism (SNP) discovery in duplicated genomes: intron-primed exon-crossing (IPEC) as a strategy for avoiding amplification of duplicated loci in Atlantic salmon (Salmo Salar) and other salmonid fishes. BMC Genomics. 2006;7:192-2.

37. Konczal M, Koteja P, Stuglik MT, Radwan J, Babik W. Accuracy of allele frequency estimation using pooled RNA-Seq. Mol Ecol Resour. 2014;14(2):381-92.

38. Dixon JR, Jung I, Selvaraj S, Shen Y, Antosiewicz-Bourget JE, Lee AY, Ye Z, Kim A, Rajagopal N, Xie W, et al. Chromatin architecture reorganization during stem cell differentiation. Nature. 2015;518(7539):331-6.

39. Eckersley-Maslin MA, Spector DL. Random monoallelic expression: regulating gene expression one allele at a time. Trends Genet. 2014;30(6):237-44.
40. Chen A, Wang R, Liu S, Peatman E, Sun L, Bao L, Jiang C, Li C, Li Y, Zeng Q, et al. Ribosomal protein genes are highly enriched among genes with allele-specific expression in the interspecific F1 hybrid catfish. Mol Gen Genomics. 2016;291(3):1083-93.

41. Deng Q, Ramskold D, Reinius B, Sandberg R. Single-cell RNA-seq reveals dynamic, random monoallelic gene expression in mammalian cells. Science. 2014;343(6167):193-6.

42. Piskol R, Ramaswami G, Li JB. Reliable identification of genomic variants from RNA-seq data. Am J Hum Genet. 2013;93(4):641-51.

43. Danzmann RG, Kocmarek AL, Norman JD, Rexroad CE, Palti Y. Transcriptome profiling in fast versus slow-growing rainbow trout across seasonal gradients. BMC Genomics. 2016;17

44. Wringe BF, Devlin RH, Ferguson MM, Moghadam HK, Sakhrani D, Danzmann RG. Growth-related quantitative trait loci in domestic and wild rainbow trout (Oncorhynchus Mykiss). BMC Genet. 2010;11

45. Gotz S, Garcia-Gomez JM, Terol J, Williams TD, Nagaraj SH, Nueda MJ, Robles M, Talon M, Dopazo J, Conesa A. High-throughput functional annotation and data mining with the Blast2GO suite. Nucleic Acids Res. 2008;36(10):3420-35

46. Salem M, Kenney PB, Rexroad CE 3rd, Yao J. Proteomic signature of muscle atrophy in rainbow trout. J Proteome. 2010;73(4):778-89.

47. Salem M, Kenney PB, Rexroad CE III, Yao J. Development of a 37 k highdensity oligonucleotide microarray: a new tool for functional genome research in rainbow trout. J Fish Biol. 2008;72(9):2187-206.

48. Rescan PY, Montfort J, Ralliere C, Le Cam A, Esquerre D, Hugot K. Dynamic gene expression in fish muscle during recovery growth induced by a fasting-refeeding schedule. BMC Genomics. 2007;8

49. Kanehisa M, Goto S, Sato Y, Furumichi M, Tanabe M. KEGG for integration and interpretation of large-scale molecular data sets. Nucleic Acids Res. 2012;40(Database issue):D109-14.

50. Fuentes EN, Ruiz P, Valdes JA, Molina A. Catabolic signaling pathways, atrogenes, and ubiquitinated proteins are regulated by the nutritional status in the muscle of the fine flounder. PLoS One. 2012;7(9):e44256.

51. Malila Y, Carr KM, Ernst CW, Velleman SG, Reed KM, Strasburg GM. Deep transcriptome sequencing reveals differences in global gene expression between normal and pale, soft, and exudative turkey meat. J Anim Sci. 2014:92(3):1250-60.

52. Fan H, Wu Y, Zhou X, Xia J, Zhang W, Song Y, Liu F, Chen Y, Zhang L, Gao $X$, et al. Pathway-based genome-wide association studies for two meat production traits in Simmental cattle. Sci Rep. 2015;5:18389.

53. Salem M, Manor ML, Aussanasuwannakul A, Kenney PB, Weber GM, Yao J. Effect of sexual maturation on muscle gene expression of rainbow trout: RNA-Seq approach. Physiol Rep. 2013;1(5):e00120.

54. Aussanasuwannakul A, Kenney PB, Weber GM, Yao JB, Slider SD, Manor ML, Salem M. Effect of sexual maturation on growth, fillet composition, and texture of female rainbow trout (Oncorhynchus Mykiss) on a high nutritional plane. Aquaculture. 2011;317(1-4):79-88.

55. AOAC. Official methods of analysis15th ed in. Washington, DC: Edited by Chemists AoOA; 1990

56. Salem M, Paneru B, Al-Tobasei R, Abdouni F, Thorgaard GH, Rexroad CE, Yao J. Transcriptome assembly, gene annotation and tissue gene expression atlas of the rainbow trout. PLoS One. 2015;10(3):e0121778.

57. Li H, Handsaker B, Wysoker A, Fennell T, Ruan J, Homer N, Marth G, Abecasis G, Durbin R. Genome project data processing S: the sequence alignment/map format and SAMtools. Bioinformatics. 2009;25(16):2078-9.

58. Kofler R, Pandey RV, Schlotterer C. PoPoolation2: identifying differentiation between populations using sequencing of pooled DNA samples (pool-Seq). Bioinformatics. 2011;27(24):3435-6.

59. Paulson TG, Galipeau PC, Reid BJ. Loss of heterozygosity analysis using whole genome amplification, cell sorting, and fluorescence-based PCR. Genome Res. 1999:9(5):482-91.

60. C HD: Chi-square test: analysis of contingency tables. In: International encyclopedia of statistical science. Edited by M L: Springer; 2011: 250-252.

61. Van der Auwera GA, Carneiro MO, Hartl C, Poplin R, Del Angel G, LevyMoonshine A, Jordan T, Shakir K, Roazen D, Thibault J et al: From FastQ data to high confidence variant calls: the Genome Analysis Toolkit best practices pipeline. Curr Protoc Bioinformatics 2013, 43:11 10 11-33.

62. O'Connell JR, Weeks DE. PedCheck: a program for identification of genotype incompatibilities in linkage analysis. Am J Hum Genet. 1998;63(1):259-66.

63. Al-Tobasei R, Paneru B, Salem M. Genome-wide discovery of long noncoding RNAs in rainbow trout. PLoS One. 2016;11(2):e0148940. 